\title{
Plantas medicinais com ação antiparasitária: conhecimento tradicional na etnia Kantaruré, aldeia Baixa das Pedras, Bahia, Brasil
}

SANTOS-LIMA, T.M.1*; SANTOS, D.R.V.2; SOUZA, R.M.2; BASTOS, N.G. ${ }^{3}$; VANNIER-SANTOS, M.A.4; NUNES, E.S.2; DIAS-LIMA, A.G. ${ }^{5}$

1 Universidade do Estado da Bahia, Programa de Pós-Graduação em Ecologia Humana e Gestão Socioambiental,, Campus VIII, Rua das Gangorras, n503, Paulo Afonso/BA, CEP: 48608-240 2Universidade do Estado da Bahia, Campus VIII, Departamento de Educação, Rua do Bom Conselho, n179, Alves de Souza, Paulo Afonso/BA, CEP:48608-230; ' Universidade do Estado da Bahia, Campus II, Departamento de Ciências Exatas e da Terra, Rodovia Alagoinhas/Salvador, $\mathrm{Br}$ 110, Km 03, Alagoinhas/BA, CEP: 48040-210; "Fundação Oswaldo Cruz, Centro de Pesquisa Gonçalo Moniz, Laboratório de Biomorfologia Parasitária, Rua Waldemar Falcão, n121, Candeal, Salvador/BA, CEP:40296-710; ' Universidade do Estado da Bahia, Campus I, Departamento Ciências da Vida, Rua Silveira Martins, n2555, Cabula, Salvador/BA, CEP: 41150-000. *Autor para correspondência: thaymacedo@hotmail.com

\begin{abstract}
RESUMO: O uso de espécies vegetais para curar doenças e sintomas remonta ao início da civilização. Em várias culturas produtos botânicos eram empregados para essa finalidade. No Brasil, sob influência das interações culturais entre índios, negros e portugueses, essa relação homem-natureza permitiu a disseminação da sabedoria herdada em relação ao uso e cultivo de diversas espécies vegetais. O presente trabalho objetivou realizar um levantamento das plantas medicinais indicadas pelos índios da etnia Kantaruré, aldeia Baixa das Pedras com ação antiparasitária. Para a coleta de dados foram realizadas entrevistas semiestruturadas com quatorze pessoas, pertencentes a uma população de 150 indígenas, selecionadas pela técnica da bola de neve, reconhecidas pela comunidade como maiores detentores do conhecimento sobre a realidade local e sobre plantas. Os resultados indicam que doze espécies são utilizadas na medicina tradicional local com ação antiparasitária, podendo destacar a caçatinga (Croton argyrophylloides Muell. Arg.), mastruz (Chenopodium ambrosioides L.), hortelã miúdo (Mentha piperita L.) e babosa (Aloe vera (L.) Burm f.) como as mais indicadas. As plantas citadas pertencem à vegetação nativa, sendo que as espécies cultivadas são encontradas principalmente nos quintais, nas proximidades das residências e em locais de cultivo próprio. Os dados levantados nesta pesquisa evidenciam a importância terapêutica, cultural e histórica do uso de espécies botânicas na prevenção e cura de enfermidades. A aldeia estudada depende diretamente dos recursos vegetais para as suas práticas de cura. Os resultados dessa pesquisa podem servir como base para bioprospecção bem como para seleção de espécies da caatinga para estudos futuros visando o seu uso e manejo sustentável.
\end{abstract}

Palavras-Chave: Etnobotânica, antiparasitária; enteroparasitos; medicina tradicional, Caatinga, indígenas.

\begin{abstract}
Medicinal plants with antiparasitic action: traditional knowledge of the Kantaruré ethnic group, Baixa das Pedras village, Bahia, Brazil. The use of plant species to cure diseases and symptoms dates back to the dawn of civilization. In various cultures, botanical products were employed for this purpose. In Brazil, influenced by cultural interactions between native indians, Africans, and the Portuguese, this man-nature relationship allowed the spread of inherited knowledge regarding the use and cultivation of various species of plants. The purpose of this study was to carry out a survey of medicinal plants with antiparasitic action indicated by the Kantaruré indians from the Baixa das Pedras village. For data collection, semi-structured interviews were conducted with 14 people belonging to an indigenous population of 150 , selected by the snowball technique, recognized by the community as the most knowledgeable on the local situation and plants. The results indicate that 12 species with antiparasitic action are used
\end{abstract}


in traditional local medicine, with emphasis on the caçatinga (Croton argyrophylloides Muell. Arg.), mastruz (Chenopodium ambrosioides L.), peppermint (Mentha piperita L.), and babosa (Aloe vera (L.) Burm f.) as the most suitable. These plants belong to the native vegetation, and the cultivated species are found mainly in backyards, near homes, and on cultivation sites. The data from this study demonstrate the therapeutic, cultural, and historical importance of the use of botanical species to prevent and cure diseases. The village studied depends directly on plant resources for their healing practices. The results of this study can serve as a basis for bioprospecting as well as for the selection of caatinga species in future studies toward its use and sustainable management.

Keywords: Ethnobotany, enteroparasites; traditional medicine, caatinga, indigenous people.

\section{INTRODUÇÃO}

O uso de espécies vegetais para a cura de doenças e sintomas remonta ao início da civilização e, em várias culturas, produtos botânicos eram empregados para essa finalidade (Halberstein, 2005; Luca et al, 2014). No Brasil, sob influência das interações culturais entre índios, negros e portugueses, essa relação homem-natureza permitiu a disseminação da sabedoria herdada em relação ao uso e cultivo de diversas espécies vegetais (Almassy et al., 2005; Liporacci \& Simão 2013).

O estudo das inter-relações diretas entre seres humanos e plantas é definido como etnobotânica (Ford, 1978; Oliveira et al 2009). É um estudo das sociedades humanas, passadas e presentes, e suas interações ecológicas, genéticas, evolutivas, simbólicas e culturais com as plantas. É praticada por muitos cientistas que a valorizam e a reconhecem como tendo um papel relevante no desenvolvimento dos povos (Hamilton et al, 2003).

A importância das plantas medicinais tem sido revelada por estudos etnobotânicos realizados em diversas regiões no Brasil, uma vez que seu território abriga uma das floras mais ricas do planeta, aliado ao fato de que o país apresenta uma alta diversidade cultural (Gottlieb et al., 1998; Toledo, 2001; Gomes \& Bandeira, 2012). Nesse contexto destaca-se o bioma Caatinga, que constitui um rico ecossistema exclusivamente brasileiro, com grande diversidade de espécies e elevada incidência de endemismo. Em recente levantamento florístico de todo o território brasileiro, o bioma caatinga apresentou o total de 4.322 espécies de plantas com sementes, sendo 744 endêmicas deste bioma, o que corresponde a $17,2 \%$ do total de táxons registrados (Forzza et al., 2012; Cordeiro \& Felix, 2013).

As plantas medicinais, como recurso terapêutico, despertam o interesse para as investigações no intuito de desenvolver fitoterápicos (Bittencourt et al, 2002). Além disso, de documentar as plantas, compreender como elas são percebidas nas comunidades, identificar a importância delas no contexto da medicina tradicional e de sua incorporação no setor de saúde pública, principalmente na Atenção Primária à Saúde no âmbito do Sistema único de Saúde (SUS) (Pires et al, 2014).

Para muitas comunidades indígenas plantas medicinais representam uma importante ferramenta na promoção da saúde (Coutinho et al, 2002), devido a questões históricas, culturais e do ambiente onde a maioria desses povos vive, essa relação se torna mais estreita. No geral, passam seus conhecimentos através de informações não registradas, transmitidas no espaço e tempo, e via de regra, através da comunicação oral (Toledo \& Barrera-Bassols, 2010; Luca et al, 2014).

Para Pilla et al (2006), ao resgatar este conhecimento e suas técnicas terapêuticas temse uma maneira de deixar registrado um modo de aprendizado informal que pode vir a contribuir para uma melhor valorização da medicina popular e, como consequência, gerar informações sobre a saúde da comunidade local.

Uma característica marcante da grande maioria das áreas indígenas é a precariedade das condições de saneamento. A maioria das residências, postos de saúde e escolas possuem infraestrutura sanitária inadequada (Santos \& Coimbra Jr, 2005). É comum também a ausência de infraestrutura destinada à coleta dos dejetos e a inexistência de água potável nas aldeias, sendo esse fator culminante para a disseminação de parasitoses gastrointestinais.

O maior quantitativo dos trabalhos realizados sobre o uso de plantas medicinais aborda o tratamento de doenças no geral, sendo que, o uso de plantas com ação antiparasitária é encontrado como dados secundários nessas pesquisas. Desse modo, o presente estudo teve como principal objetivo realizar um levantamento das plantas indicadas na medicina tradicional em uma aldeia indígena na região Nordeste do Brasil, utilizadas para o tratamento de doenças causadas por parasitos gastrointestinais. 


\section{MATERIAL E MÉTODO \\ Área de estudo}

$O$ estudo foi realizado na terra indígena Kantaruré, aldeia Baixa das Pedras, pertencente ao município de Glória, situado da região nordeste da Bahia, localizado a $514 \mathrm{~km}$ de Salvador, capital do Estado, com área de $1.255,647 \mathrm{~km}^{2}$. O município apresenta 16.000 habitantes, distribuídos entre as áreas urbana e rural, com a presença de diversas etnias indígenas. O clima é semiárido com chuvas de fevereiro a abril e alto risco de seca, sendo a temperatura média anual de $25^{\circ} \mathrm{C}$, e a vegetação predominante é a do tipo caatinga.

A aldeia fica situada a uma distância de 43 km do município de Paulo Afonso, próxima à vertente setentrional da Serra Grande, a aproximadamente $8 \mathrm{~km}$ da margem direita do Rio São Francisco. Ocupa uma área de 1.811 hectares, homologada em 2001, habitada por cerca de 150 índios e distribuídos em aproximadamente 45 famílias, segundo dados do último censo do IBGE (2010).

\section{Coleta e análise de dados}

Para a coleta de dados, inicialmente, foram contatadas as lideranças indígenas responsáveis, para esclarecimento dos objetivos do trabalho e para obter permissão para o desenvolvimento da pesquisa, com assinatura do Termo de Consentimento Livre e Esclarecido. As primeiras visitas tiveram como principal objetivo conhecer a comunidade, estabelecer o contato com os moradores e identificar seus hábitos e modo de vida. Juntamente com as lideranças e agentes comunitários de saúde se caracterizou a área de estudo. Durante esse período de reconhecimento foram estabelecidos contatos prévios com as famílias para o esclarecimento sobre o trabalho a ser desenvolvido no local. A presente pesquisa foi aprovada pelo Comitê de Ética em Pesquisa (CEP) da Universidade do Estado da Bahia, sob o CAAE 11009812000000057 e número de parecer 336.845 .

A coleta de dados foi desenvolvida no período de agosto a novembro de 2014, por meio de entrevistas semiestruturadas nas quais se dialogou sobre as plantas com ação antiparasitária, com ênfase em protozoários e helmintos enteroparasitas, reconhecidas para a cura e tratamento das doenças, e as partes da planta que são utilizadas.

Para a seleção dos entrevistados que participaram nesta pesquisa, utilizou-se a técnica "bola de neve" (ou Snow Ball") (Bailey, 1994), buscando aqueles moradores, reconhecidos pela comunidade como os conhecedores locais detentores do maior acervo de conhecimento etnobotânico. A partir do contato inicial com a comunidade, um primeiro especialista é reconhecido, que passa a indicar outro especialista e assim sucessivamente, envolvendo todos os especialistas da comunidade, até que o ciclo se feche e novos especialistas não sejam mais apontados (Albuquerque et al, 2010). Para essa pesquisa foram indicados quatorze especialistas, sendo que nove homens e cinco mulheres.

Durante a realização das entrevistas foi solicitado aos entrevistados que listassem as plantas utilizadas, e que as citassem de acordo com a frequência de uso e importância. A lista livre obtida permitiu buscar informações especificas sobre um domínio cultural da comunidade estudada, como também estimar as plantas que sofrem grande pressão de uso pela população na área estudada (Bernard, 1988; Weller \& Romney, 1988; Gomes \& Bandeira, 2012). Com a listagem, pode-se observar as espécies culturalmente relevantes, pois faz inferências sobre a estrutura perceptiva do informante a partir da ordem em que o mesmo recorda-se e coloca os elementos em dada ordem.

A partir da ordenação das espécies feita por cada informante, os dados são convertidos em um ranking e divididos pelo número total de itens em cada lista livre, atribuindo-se um Valor de Saliência (S) para cada item (Gomes \& Bandeira, 2012). Para tabulação dos dados utilizou-se o programa ANTROPAC 9.48, que calcula o somatório de saliência das espécies em comum de todas as listas livres.

Após a realização das entrevistas foram feitas visitas aldeia para a coleta do material botânico com o auxílio da liderança local, objetivando o reconhecimento das espécies in loco. As amostras foram coletadas e processadas de acordo com a metodologia proposta por Mori et al (1989). O material coletado foi depositado no Herbário da Universidade do Estado da Bahia (HUNEB), Campus II Alagoinhas e identificados com ajuda de especialistas. Para a classificação das famílias botânicas foi utilizado o sistema APG III (2009).

Algumas das espécies indicadas não foram coletadas devido ao clima local, com altas temperaturas e chuvas escassas. Em decorrência da irregularidade na variação da precipitação pluviométrica anual, as espécies sofrem variação fenológica e nem sempre conseguem produzir flores e frutos. A determinação da nomenclatura botânica dessas plantas foi realizada por comparação, e da avaliação de outros trabalhos que se utilizam da nomenclatura local em seus resultados desenvolvidos na mesma região no estado do Nordeste. Para a confirmação da nomenclatura botânica, foram consultadas bibliografias especializadas além dos sites de indexação de plantas IPNI (The Internacional Plant Names Index) e Flora do Brasil. 


\section{RESULTADOS E DISCUSSÃO}

Foram entrevistadas quatorze pessoas, sendo nove homens e cinco mulheres, que citaram 12 nomes de plantas potencialmente utilizadas como medicinais com ação antiparasitária, correspondendo a doze espécies e doze famílias diferentes. Nesse estudo foi possível observar que os homens foram indicados como maiores detentores do conhecimento (Figura 1). Resultado não comum na literatura, como nos trabalhos de Pires et al (2014), Rodrigues \& Andrade (2014) e Oliveira (2013), nos quais a mulher tem um papel de destaque acerca do saber, cultivo e preparo das plantas medicinais. Os dados referentes aos recursos vegetais utilizados com ação antiparasitária, indicados nessa pesquisa estão sistematizados na Tabela 01.

A família que teve maior número de indicações foi a Euphorbiaceae, representada pela caçatinga (Croton argyrophylloides Muell. Arg) com nove indicações, seguida por Lamiaceae, representada pelo hortelã miúdo (Mentha piperita L.) com cinco indicações. A família Xanthorrhoeaceae e Amaranthaceae, representadas pela babosa (Aloe vera (L.) Burm f) e mastruz (Chenopodium ambrosioides L.) respectivamente, tiveram quatro indicações cada. As famílias Olacaceae, Caesalpinioideae e Myrtaceae, representadas pela ameixa silvestre (Ximenia americana L.), catingueira (Poincianella pyramidalis (Tul.) L.P.Queiroz var. pyramidalis) e goiabeira (Psidium guajava L.) com três indicações. Com duas indicações cada, ficaram as famílias Verbenaceae, Liliaceae, Anacardiaceae e a Caricaceae, representadas pelo alecrim (Lippia thymoides Mart. \& Schauer), alho (Allium sativum L.), cajueiro (Anacardium occidentale L.) e mamão (Carica papaya L.) respectivamente. E com uma indicação a família Musaceae, representado pela banana (Musa paradisiaca L.).

A diversidade de famílias botânicas encontradas no estudo é semelhante a trabalhos como o de Coutinho et al (2002) e Colaço (2009) desenvolvidos em comunidades indígenas do Nordeste. Onde todas as famílias botânicas encontradas nesse estudo são encontradas nos trabalhos dos autores citados.

Dentre as espécies que tiveram maior frequência, a caçatinga teve um maior número de citações. Nas pesquisas realizadas por Bandeira et al (2003), Colaço (2009) e Modercin (2010), a espécie é indicada como planta medicinal, mas em nenhum desses o uso é destacado como ação antiparasitária. Já foram realizados alguns estudos que comprovaram a eficácia em várias ações biológicas de outras espécies do gênero Croton, como antimicrobiano (Albuquerque et al., 1974), antioxidante (Catunda Júnior et al., 2002) e agente larvicida (Lima et al., 2006).

A descrição da composição química comprovando a ação antiparasitária da caçatinga Croton argyrophylloides Muell. Arg), não foi encontrada em literatura. Com isso, há uma necessidade de pesquisas para testar seu potencial biológico.

O uso da hortelã miúdo com ação antiparasitária foi citado no estudo de Gomes \& Bandeira (2012), onde se pode observar uma alta frequência de citação, podendo ser comparado a pesquisa aqui realizada (segunda espécie com maior número de citações). Outros estudos como o de Costa \& Mayworm (2011) e Battisti et al (2013) apontam a hortelã miúdo com ação antiparasitária e também demonstram alta frequência de indicações

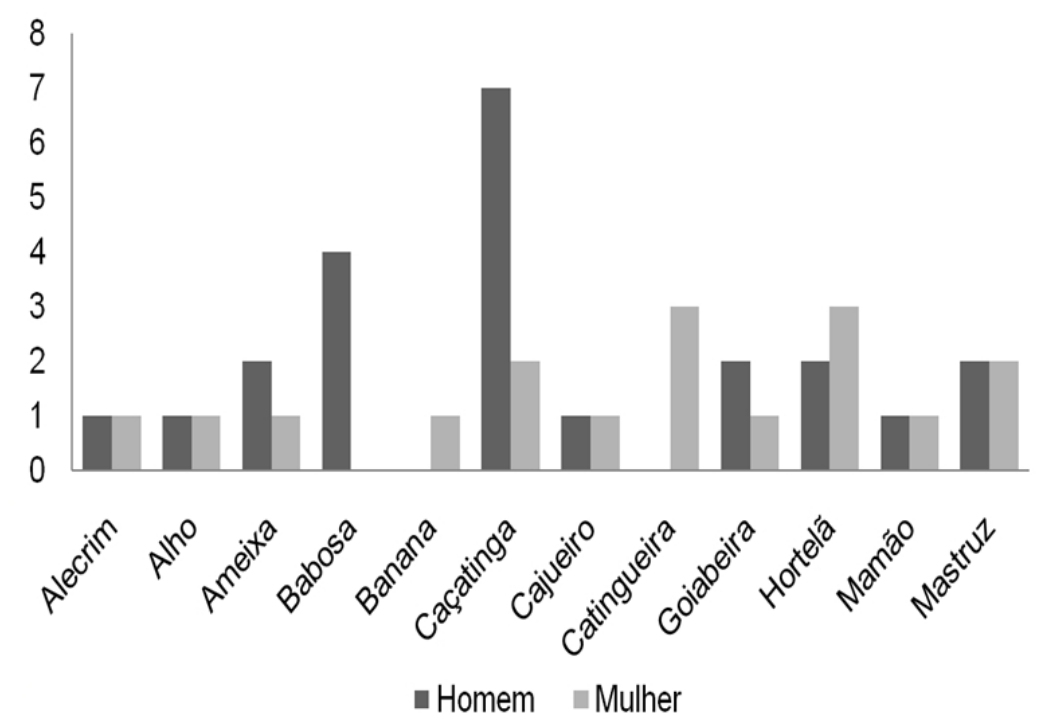

FIGURA 1. Número de espécies citadas por gênero com ação antiparasitária, na aldeia indígena Baixa das Pedras, etnia Kantaruré, Bahia.

Rev. Bras. PI. Med., Campinas, v.18, n.1, supl. I, p.240-247, 2016. 
em seus trabalhos.

Em experimento realizado por Adjuto (2008), a folha do hortelã miúdo apresentou uma concentração elevada do óxido de piperitenona, elemento que elimina amebas e giárdias, parasitas intestinais. Segundo este autor, o chá feito em alta concentração tem o mesmo efeito dos remédios encontrados na farmácia.

A babosa, uma das plantas que teve um número considerável de citações nessa pesquisa, foi indicada como sendo de ação antiparasitária em alguns trabalhos como o de Coutinho et al (2002), Castro (2006), Gomes \& Bandeira (2012) e Battisti et al (2013), os quais teve alta frequência de citações, indicando que a mesma tem alta representatividade medicinal. A folha da babosa é rica em aloína, um vermífugo que elimina os protozoários que parasitam o intestino (Atherton, 1997).

O mastruz foi uma das espécies mais citadas nos trabalhos sobre plantas medicinais com ação antiparasitária. Paiva \& Neves (2009), Gomes \& Bandeira (2012), Oliveira et al (2013) em seus respectivos trabalhos destacaram o mastruz com uma das espécies mais indicadas. Nesses trabalhos, a planta teve grande destaque, sendo incluída também como terapêutica para outras enfermidades.

A atividade anti-helmíntica desta planta tem sido atribuída ao ascaridol, constituinte predominante do óleo essencial. No entanto, este óleo não tem sido freqüentemente utilizado na medicina humana, devido a sua toxicidade para mamíferos (Macdonald et al., 2004; Silva, 2012). As propriedades biológicas do mastruz vêm, no entanto, sendo investigadas em extratos da planta, incluindo o extrato aquoso (Almeida et al., 2007; Silva, 2012). Resultados de estudo in vitro demonstram que tanto o ascaridol, quanto a infusão das folhas isentas de ascaridol, apresentaram atividade anti-helmíntica, sugerindo que possivelmente, outros constituintes presentes na planta, que não o ascaridol, mas sim componentes hidrofílicos também estejam relacionados com esta atividade (MacDonald et al., 2004; Silva, 2012).

O alho em experimentos realizados por Ankiri et al, 1999, Martins et al (2000) e Lima (2011), teve seu efeito antiparasitario comprovado. Pesquisa realizada por Lun et al (1994) demonstraram que a ação do composto ajoeno (ajocisteína) e dialliltrisulfeto inibem o metabolismo ou crescimento dos parasitos.

Outras plantas citadas, como a catingueira, goiabeira, alecrim, cajueiro, mamão, ameixa e a banana, são citadas em trabalhos como o de Albuquerque \& Andrade (2002). O autor destacou em seu estudo o uso de recursos vegetais da caatinga, sendo essas plantas indicadas para uso medicinal na pesquisa.

A folha foi a parte mais citada no preparo dos remédios caseiros com $58 \%$ dos usos, seguido por raiz e fruto com $16 \%$ cada, e a flor com $8 \%$. A forma de uso mais comum foi o chá (decocção ou infusão) com $83 \%$ das indicações, e a maceração com 17\%. Pasa \& Ávila (2010) ao realizar um trabalho etnobotânico verificaram que o uso das folhas para preparo dos medicamentos resultou

TABELA 1. Espécies citadas pelos entrevistados na aldeia indígena Baixa das Pedras, etnia Kantaruré, Bahia,

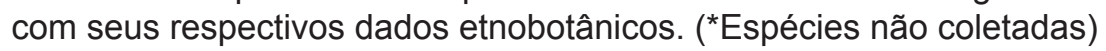

\begin{tabular}{|c|c|c|c|c|c|}
\hline $\begin{array}{l}\text { Nome popularl } \\
\text { Voucher number }\end{array}$ & Espécie & Família & $\begin{array}{c}\text { Parte } \\
\text { utilizada }\end{array}$ & $\begin{array}{c}\text { Formas de } \\
\text { uso }\end{array}$ & Saliência \\
\hline Caçatinga (14379) & Croton argyrophylloides Muell. Arg. & Euphorbiaceae & Raiz & Chá (Infusão) & 0,480 \\
\hline Mastruz (14390) & Chenopodium ambrosioides L. & Amaranthaceae & Folha & Chá & 0,274 \\
\hline Hortelã miúdo $(\mathrm{NC})^{*}$ & Mentha piperita L. & Lamiaceae & Folha & Chá & 0,22 \\
\hline Babosa (14388) & Aloe vera (L.) Burm f. & Xanthorrhoeaceae & Folha & Chá & 0,193 \\
\hline Alho $(\mathrm{NC})^{*}$ & Allium sativum $L$. & Liliaceae & Raiz & $\begin{array}{l}\text { Chá (Infusão); } \\
\text { Maceração }\end{array}$ & 0,143 \\
\hline Goiabeira (14377) & Psidium guajava L. & Myrtaceae & Folha & Chá & 0,143 \\
\hline Ameixa silvestre $(\mathrm{NC})^{*}$ & Ximenia americana L. & Olacaceae & Folha & Chá & 0,102 \\
\hline Mamão (14385) & Carica papaya L. & Caricaceae & Fruto & Maceração & 0,095 \\
\hline Cajueiro (14373) & Anacardium occidentale L. & Anacardiaceae & Folha & Chá & 0,076 \\
\hline Catingueira (14376) & $\begin{array}{c}\text { Poincianella pyramidalis (Tul.) L.P.Queiroz var. } \\
\text { pyramidalis }\end{array}$ & Caesalpinioideae & Flor & Chá & 0,071 \\
\hline Alecrim (14374) & Lippia thymoides Mart. \& Schauer & Verbenaceae & Folha & Chá & 0,048 \\
\hline Banana (14383) & Musa paradisíaca $L$. & Musaceae & Fruto & Maceração & 0,048 \\
\hline
\end{tabular}

Rev. Bras. PI. Med., Campinas, v.18, n.1, supl. I, p.240-247, 2016. 
em maior utilização. Resultado também obtido por Oliveira et al (2013), onde a utilização de folha correspondeu a $46 \%$ das indicações na pesquisa. Os resultados apontados são inferiores ao dessa pesquisa, onde a folha teve $58 \%$ de indicação para uso. A folha é considerada a parte mais usada principalmente para a categoria medicinal, visto que a maioria dos princípios ativos existentes nas plantas podem ser encontrados nessa parte.

Os resultados para o valor de saliência (Tabela 1) das espécies utilizadas como medicinais variou entre 0,04 a 0,48 . As seis espécies que tiveram maior valor de saliência cultural, em ordem crescente foram: ameixa silvestre (Ximenia americana L. - 0,10), goiabeira (Psidium guajava L. - 0,14), babosa (Aloe vera (L.) Burm f. - 0,19), mastruz (Chenopodium ambrosioides L. - 0,27), hortelã miúdo (Mentha piperita L. - 0,22) caçatinga (Croton argyrophylloides Muell. Arg - 0,48).

Das seis plantas que tiveram maior frequência de citação, três delas (hortelã miúdo, babosa e ameixa silvestre) apresentaram maior valor de saliência. Essa diferença se dá por conta do ordenamento em que as plantas foram citadas (ranking), assim as espécies que se mostraram mais salientes culturalmente estão entre as espécies que também são mais frequentes.

Almeida \& Albuquerque (2002) e Gomes \& Bandeira (2012) corroboram que os dados obtidos em pesquisas dessa natureza estão diretamente relacionados à maiorias dos casos de doenças na população. Nesse estudo, isso pode ser confirmado através de comunicação pessoal com os membros do serviço de saúde local, ao afirmarem que existe uma grande incidência de doenças causadas por parasitos intestinais. Esse quadro epidemiológico pode ser o reflexo da falta de saneamento básico e cuidados com a higiene pessoal, já que mesmo tendo água encanada essa por sua vez não possuí nenhum tipo de tratamento que possibilite um consumo seguro para a saúde local.

Os resultados encontrados vêm indicar que é de suma importância o registro dos conhecimentos tradicionais e práticas de cura das populações indígenas, que podem vir futuramente nortear a criação de políticas públicas de saúde mais eficazes para essas populações. Essa pesquisa também demonstra que os resultados servem como base para bioprospecção e validação a partir de estudos farmacobotânicos capazes de isolar as substâncias bioativas que atuam no tratamento ou erradicação dos sinais, bem como para seleção de espécies da caatinga para estudos futuros visando o seu uso e manejo sustentável.

\section{AGRADECIMENTOS}

À etnia indígena Kantaruré pelo acolhimento, receptividade, colaboração e contribuição que foram de fundamental importância para a realização desta pesquisa. Ao Programa de Mestrado em Ecologia Humana e Gestão Socioambiental, ao qual esse projeto está vinculado. À Coordenação de Aperfeiçoamento de Pessoal de Nível Superior, CAPES, pela bolsa de estudos que foi de fundamental importância durante o decorrer da pesquisa. E a todas as pessoas que direta ou indiretamente contribuíram para o desenvolvimento do trabalho.

\section{REFERÊNCIAS}

ADJUTO, E.N.P. Caracterização morfológica e de óleo essencial de seis acesos de Hortelanzinho (Mentha spp.). 2008. 79p. Dissertação (Mestrado - Área de concentração Ciências Agrárias) - Departamento de Agronomia e Medicina Veterinária, Universidade de Brasília, Brasília.

ALBUQUERQUE, U.P.; ANDRADE, L.H.C. Uso de recursos vegetais da caatinga: $O$ caso do agreste do estado de Pernambuco (nordeste do Brasil). Interciencia, v.27, n.7, 336-345, 2002.

ALBUQUERQUE, U.P. et al. Métodos e Técnicas na pesquisa Etnobiológica e Etnoecológica. $2^{a}$. ed. Recife: NUPEEA, 2010.559p

ALBUQUERQUE, M. et al. Antimicrobial substances of superior plants. Isolation of diterpene acid from Croton aff. Argyrophylloides Muell. Arg. Euphorbiaceae. Revista do Instituto de Antibiótico, v.44, n.14, 8389, 1974.

ALMASSY, J.A.A.; LOPES, R.C; ARMOND, C.; SILVA, F.; CASALI, V.W.D. Folhas de chá: Plantas Medicinais na Terapêutica Humana. 1.ed. Viçosa: UFV, 2005. 233p.

ALMEIDA, C.F.C.B.R.; ALBUQUERQUE, U. P. Uso e conservação de plantas e animais medicinais no estado de Pernambuco (Nordeste do Brasil): um estudo de caso. Interciência, v.27, n.6, p.276-285, 2002.

ALMEIDA, M.A.O. et al. Efeitos dos extratos aquosos de folhas de Mentha piperita L. e de Chenopodium ambrosiodes L. sobre cultivos de larvas infectantes de nematódeos gastrintestinais de caprinos. Revista Brasileira de Parasitologia Veterinária, v.16, n.1, p.57-59, 2007.

ANKIRI, S.; MIRELMAN, D. Antimicrobial properties of allicin from garlic. Microbes Infection, v.1, n.2, p. 125129, 1999.

APG III. Au update of the angiosperm Phylogeny Group classification for the orders and families of flowering plants: APG III. Botanical Journal of the Linnean Society, v.161, p.105-121, 2009.

ATHERTON, P. Aloe vera revisited. The British Journal of Phytotherapy, v.4, n.4, p.176-183,1997.

BAILEY, K. Methods of Social Research. 4ed. New York: The Free Press, 1994, 588p.

BANDEIRA, F.P.S.; DANTAS, M.F.B.; CHAVES, J.M.; MACHADO, C.G. Diagnóstico Etnoambiental Pankararé. 1.ed. Feira de Santana: UEFS, 2003.

BATTISTI, C. et al. Plantas medicinais utilizadas no município de Palmeira das Missões, RS Brasil. Revista Brasileira de Biociências, v. 11, n.3, p. 338-348, 2013.

Rev. Bras. PI. Med., Campinas, v.18, n.1, supl. I, p.240-247, 2016. 
BERNARD, H.R. Research methods in cultural anthropology. 2.ed. USA: Sage Publications, 1988, $520 p$.

BITTENCOURT, S.C. et al. O uso das plantas medicinais sob prescrição médica: pontos de diálogo e controvérsias com uso popular. Revista Brasileira de Farmacognosia, v. 12, supl., p.89-91, 2002.

CASTRO, D.L.L. Aspectos toxicológicos das plantas medicinais utilizadas no Brasil: um enfoque qualitativo no Distrito Federal. 2006. 63p. Monografia (Especialização - Área de concentração em Qualidade de Alimentos) - Centro de Excelência em Turismo, Universidade de Brasília, Brasília.

CATUNDA JUNIOR, F.E.A. et al. Atividade antioxidante de óleo essenciais de plantas do nordeste do Brasil. Ciência e tecnologia, v. 4, p. 23-29, 2002.

COLAÇO, M.A.S. Etnobotânica dos Índios Pankararé no Raso da Catarina - Bahia: Uso e Importância Cultural de Plantas da Caatinga. 2009. $105 \mathrm{f}$. Dissertação (Mestrado em Botânica) Departamento de Ciências Biológicas, Universidade Estadual de Feira de Santana, Feira de Santana.

CORDEIRO, J.M.P.; FÉLIX, L.P. Conhecimento botânico medicinal sobre espécies vegetais nativas da caatinga e plantas espontêneas no agreste da Paraíba, Brasil. Revista Brasileira de Plantas Medicinais, v.16, n.3, supl. I, p.685-692, 2013.

COSTA, V.P.; MAYWORM, M.A.S. Plantas medicinais utilizadas pela comunidade do bairro dos Tenentes município de Extrema, MG, Brasil. Revista Brasileira de Plantas Medicinais, v.13, n.3, p.282-292, 2011.

COUTINHO, D.F. et al. Estudo etnobotânico de plantas medicinais utilizadas em comunidades indígenas no estado do Maranhão - Brasil. Visão Acadêmica, v.3, n.1, p. 7-12, 2002.

FORD, R.I. Ethnobotany: historical diversity and synthesis. In: R. I. Ford (Ed). The nature and status of etnobotany. 1 ed. Michigan: Anthopological Papers, 1978, p.33-49.

FORZZA, R.C. et al. New Brazilian Floristic List Highlights Conservation challenges. BioScience, v.62, n.1, p 3945, 2012.

GOMES, T.B.; BANDEIRA, F.P.S.F. Uso e diversidade de plantas em uma comunidade quilombola no Raso da Catarina, Bahia. Acta Botânica Brasilica, v.26, n.4, p. 796-809, 2012.

GOTTLIEB, S.L. et al. Biodiversidade: o enfoque interdisciplinar brasileiro. Ciência e Saúde Coletiva, v.3, n.2, p. 97-102, 1998.

HALBERSTEIN, R.A. Medicinal Plants: Historical and Cross-cultural Usage Patterns. Medicinal Plant Usage, n.15, p.686-699, 2005.

HAMILTON, A.C. et al. The purposes and teaching of Applied Ethnobotany. Godalming, People and Plants working paper, v.11, p. 1-71, 2003.

IBGE Censo Demográfico 2000 e 2010. 2010. Disponível em: <http://www.ibge.gov.br/indigenas/indigena_ censo2010.pdf >. Acesso em 20 agos. 2014.

LIMA, C.M.B.L. Investigação da atividade antiparasitária do Allium sativum L. in vitro e in vivo. 2011. 112p. Tese (Doutorado - Área de concentração Produtos Naturais e Sintéticos Bioativos) - Departamento de Farmacologia, Universidade Federal da Paraíba,
Paraíba.

LIMA, M.G.A. et al. Efeito dos extratos de caule e folha de espécies de Euphorbiaceae sobre larvas de Aedes aegypti (Diptera, Culiadae). Revista do instituto de Medicina Tropical de São Paulo, v48, n.4, p.211-214, 2006.

LIPORACCI, H.S.N.; SIMÃO, D. G. Levantamento etnobotânico de plantas medicinais nos quintais do Bairro Novo Horizonte, Ituiutaba, MG. Revista Brasileira de Plantas Medicinais, v.15, n.4, p.529540, 2013.

LUCA, V.D. et al. Utilização de plantas medicinais no entorno do Parque Estadual da Serra Furada, Santa Catarina, Brasil: uma abordagem etnobotânica. Revista Brasileira de Biociências, v. 12, n.2, p. 59-65, 2014.

LUN, Z.R. et al. Antiparasitic activity of diallyl trisulfide (Dasuansu) on human and animal pathogenic protozoa (Trypanossoma sp., Entamoeba Histolytica and Giardia lamblia) in vitro. Annales de la Societe Belge de Medecine Tropicale, v.74, n.1, p.51-59, 1994.

MACDONALD, D. et al. Asacaridole-less infusions of Chenopodium ambrosioides contain a nematocide(s) that is(are) not toxic to mammalian smooth muscle. Journal Ethnopharmacol, v.92, p.215-221, 2004.

MARTINS, E.R.; CASTRO, D.M.; CASTELLANI, D.C.; DIAS, J.E. Plantas Medicinais. 1.ed. Viçosa: UFV, 2000. 220p.

MODERCIN, I.F. Rancho do Jatobá do meio do mundo: Etnografia da agricultura Pankararé e a relação dos índios com o ambiente. 2010. 193p. Dissertação (Mestrado Área de Concentração Antropologia) - Departamento de Antropologia, Universidade Federal da Bahia,Bahia.

MORI, S.A., SILVA, L.A. M.; LISBOA, G., CORADIN, L. Manual de manejo do herbário fanerogâmico. 24.ed. Bahia: Centro de Pesquisa do Cacau, 1989. 104p.

PASA, M.C.; ÁVILA, G. Ribeirinhos e recursos vegetais: a etnobotânica em Rondonópolis, Mato Grosso, Brasil. Interações, v. 11, n.2, p. 195-204, 2010.

PAIVA, L.J.M., NEVES, M. F. Controle Orgânico de Parasitas. Revista Científica Eletrônica de Medicina Veterinária, v. 12, p.1-8, 2009.

PILLA, M.A.C. et al. Obtenção e uso das plantas medicinais no distritode Martim Francisco, Municipio de Mogi-Mirim, SP, Brasil. Acta Botanica Brasilica, v.20, p.789-802, 2006.

PIRES, I.F.B. et al. Plantas medicinais como opção terapêutica em comunidade de Montes Claros, Minas Gerais, Brasil. Revista Brasileira de Plantas Medicinais., v. 16, n.2, supl. I, p.426-433, 2014.

OLIVEIRA, F.C. et al. Avanços nas pesquisas etnobotânicas no Brasil. Acta Botânica Brasilica. V.23, n.2, p.590605, 2009.

OLIVEIRA, W.A. Os recursos vegetais e o saber local nos quintais da comunidade de Santo Antônio do Caramujo, Cáceres, Mato Grosso, Brasil. 2013. 193p. Dissertação (Mestrado - Área de concentração Ciências Florestais e Ambientais) - Departamento de Ciências Florestais e Ambientais, Universidade Federal do Mato Grosso, Cuiabá.

RODRIGUES, A.P.; ANDRADE, L.H.C. Levantamento etnobotânico das plantas medicinais utilizadas pela comunidade de Inhamã, Pernambuco, Nordeste do Brasil. Revista Brasileira de Plantas Medicinais, v.16, 
n.3, supl. I, p.721-730, 2014.

SANTOS, R.V.; COIMBRA J.R., C.E.A. Cenários e tendências da saúde e de epidemiologia dos povos indígenas no Brasil. In: COIMBRA J.R. (ED). Epidemiologia e Saúde dos povos indígenas no Brasil. Rio de Janeiro: Editora Fiocruz, 2005. p.13-48.

SILVA, G.D. Avaliação da atividade anti-helmintica e toxicológica do extrato aquisi de Chenopodium ambrosioides (mastruz) sobre nematoides gastrointestinais de caprinos. 2012. 66p. Dissertação (Mestrado - Área de concentração Ciência animal dos Trópicos) - Departamento de Patologia e Clínicas,
Universidade Federal da Bahia, Bahia.

TOLEDO, V.M. Biodiversity and indigenous peoples. In: Levin, S.A. (Ed.). Encyclopedia of Biodiversity. San Diego: Academic Press, 2001. p. 330-340.

TOLEDO, V.M.; BARRERA-BASSOLS, N. A etnoecologia: uma ciência pós-normal que estuda sabedorias tradicionais. In: SILVA, V.A., ALMEIDA, A.L.S.; ALBUQUERQUE, U. P.(Ed.). Etnobiologia e Etnocologia: Pessoas e natureza na América Latina. Recife: Nupeea, 2010. p. 13-36.

WELLER, S.C., ROMNEY, K.A. Systematic data collection. 1.ed. California: Sage Publications, 1988. 96p.

Rev. Bras. PI. Med., Campinas, v.18, n.1, supl. I, p.240-247, 2016. 